\title{
Granulocytes: From Basic Research to Clinical Use
}

\author{
Torsten J. Schulze Peter Bugert \\ ${ }^{a}$ Institute Springe, German Red Cross Blood Donor Services NSTOB, Springe, Germany; \\ bInstitute of Transfusion Medicine and Immunology, Medical Faculty Mannheim, Heidelberg University, German Red Cross \\ Blood Service Baden-Württemberg - Hessen, Mannheim, Germany
}

Neutrophilic granulocytes (neutrophils) are the most abundant leukocytes in human blood and play a central role in the innate immune response [1]. This issue follows mature neutrophils through their various aspects in medical therapies as well as through immunological interactions against them that demand a professional response. In short, this issue sheds light on the variety of different roles that granulocytes play in the field of transfusion medicine.

It starts naturally in pregnancy, when maternal antibodies against fetal granulocytes bearing paternal antigens lead to complications in terms of loss of this part of the innate immune defense, rendering the newborns vulnerable to bacterial, viral or fungal infections. Porcelijn and de Haas [2] reflect on this antibody-dependent condition, the neonatal alloimmune neutropenia (NAIN), as well as its state-of-the-art diagnosis and treatment. Although the condition is relatively rare, knowledge about NAIN is crucial: an early diagnosis may prevent a bad outcome, and therefore physicians need to be trained in the differential diagnosis [2]. The underlying dependency on certain polymorphisms on a group of neutrophilic glycoproteins has been understood better within the last decades. These antigens now form a blood group system of its own: the human neutrophil antigens (HNA). Much progress has been made within the last years in terms of genotypic characterization, understanding of antigen structure, and discovery of new alloantibody specificities. In their review, Flesh and Reil [3] give an up-to-date comprehensive view on the HNA system.

When the mothers that have developed alloantibodies against neutrophils donate blood, the same antibodies can lead to a severe transfusion reaction, the transfusion-related acute lung injury (TRALI), a condition with a poor prognosis when untreated. By activation of resting neutrophils within the pulmonary vessels the lungs become filled with transudate. Rebetz et al. [4] summarize and discuss the similarities of acute respiratory distress syndrome (ARDS) and TRALI.

Granulocyte transfusions on the other hand may lead to a complication that can be best described as 'reverse-TRALI': Transfusion of granulocyte preparations against pre-existing antibodies against HLA class I, II or against HNA may activate all transfused neutro- phils. While these antibodies are not exclusively limited to women (after pregnancy), it is during pregnancy that the vast majority of these antibodies are being formed [5]. In theory, regarding immunization against neutrophils, and more than pregnancies or transfusion of leukocyte-reduced blood preparations, granulocyte preparations should lead to the formation of alloantibodies. Surprisingly, this does not happen very often and may be attributed to a compromised immune reaction in the setting of granulocyte substitution [6]. Be it a pre-existing antibody or antibody formation under treatment that include granulocyte preparations, screening for patients' leukocyte antibodies as well as crossmatch prior to transfusion are strongly recommended. Antibodies against neutrophils, however, are only one aspect in the context of granulocyte transfusion that can impair the therapeutic outcome. Other aspects may depend on the precise clinical situation of the patient, his treatment, the time of neutropenia, the time of sepsis, the specific underlying disease itself, the mobilizing regimen for the donor, or whether pooled or apheresis granulocyte preparations are used. To pave the way for specific studies and to establish a standard setting for granulocyte transfusion, Pagano et al. [7] present an international registry of granulocyte transfusion.

In order to find adequate donors not only for the provision of suitable platelet or red blood cell (RBC) but also for granulocyte preparations, Portegys et al. [8] present the implementation of a regional registry of blood donors extendedly typed for blood group, platelet and granulocyte antigens. Interestingly, the authors introduced the first molecular method for large-scale screening to identify donors with the HNA-2 $2^{\text {null }}$ phenotype. Once typed, the close contact of a regional blood donation center to its frequent donors may help react swiftly to a sudden demand of preparations of a certain blood group - RBCs, platelets or granulocytes - for patients with alloantibodies. With the growing of a registry the probability rises of having suitable preparations in stock.

Finally, Anyanwu et al. [9] lead over from the mentioned classical preparations towards new techniques of collecting mononuclear cells for the fast developing field of cellular immune therapies.

\section{KARGER}

(๑) 2018 S. Karger GmbH, Freiburg

Fax +497614520714 


\section{References}

1 Kobayashi SD, DeLeo FR: Role of neutrophils in innate immunity: a systems biology-level approach. Wiley Interdiscip Rev Syst Biol Med 2009;1:309-333.

2 Porcelijn L, de Haas M: Neonatal alloimmune neutropenia. Transfus Med Hemother 2018;45(5): DOI: $10.1159 / 000492949$.

3 Flesch BK, Reil A: Molecular genetics of the human neutrophil antigens. Transfus Med Hemother 2018; 45(5): DOI: 10.1159/000491031.

4 Rebetz J, Semple JW, Kapur R:. The pathogenic involvement of neutrophils in acute respiratory distress syndrome and transfusion-related acute lung injury. Transfus Med Hemother 2018;45(5): DOI: 10.1159/000492950.
Nguyen XD, Schulze TJ, Seifried E: Granulocyte antibodies in male donors: can they trigger transfusionrelated acute lung injury? Transfusion 2018;58:18941901.

6 Price TH, McCullough J, Assmann SF: WBC alloimmunization. Effects on the laboratory and clinical endpoints of therapeutic granulocyte transfusions. Transfusion 2018;58:1280-1288.

7 Pagano MB, Morton S, Cohn CS, Gross S, Kutner J, Lewin A, McCullough J, Schweitzer I, Tinmouth AT, West K, Stanworth SJ: An international registry of granulocyte transfusions. Transfus Med Hemother 2018;45(5): DOI: 10.1159/000492629.
Portegys J, Rink G, Scharberg EA: Towards a regional registry of extended typed blood donors: molecular typing for blood group, platelet and granulocyte antigens. Transfus Med Hemother 2018;45(5): DOI: $10.1159 / 000493555$.

9 Anyanwu A, Sitzmann N, Hetjens S, Klüter H, Wuchter P: Low-volume leukapheresis in non-cytokine-stimulated donors for the collection of monocytes. Transfus Med Hemother 2018;45(5): DOI: $10.1159 / 000490859$. 